\title{
Selection of Macroporous Resins for the Separation and Detection of
}

\section{Tomato Saponins}

\author{
Jinlei Liu ${ }^{1 \mathrm{a}}$, Ziming Yang ${ }^{1 \mathrm{~b}}$, Fenglai $\mathrm{Lu}^{1 \mathrm{c}}$, Sicheng Chen ${ }^{1 \mathrm{~d}}$, Dianpeng $\mathrm{Li}^{1 \mathrm{e} *}$

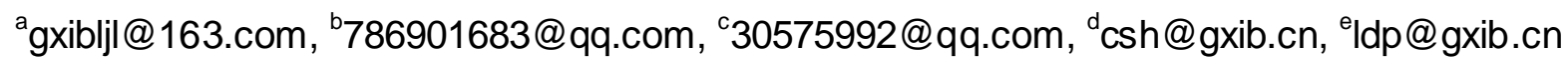

${ }^{1}$ Guangxi Key Laboratory of Functional Phytochemicals Research and Utilization, Guangxi Institute of Botany, Guilin 541006, China

Key words Tomato saponins; Macroporous resin; Adsorption and purification

Abstract This study was conducted to select a type of macroporous resin most suitable for the separation and purification of tomato saponins. The content of esculeoside $\mathrm{A}$ which is the major part of total tomato saponins was detected by colorimetry, and extraction rate of esculeoside A was used as an indicator to select the best type of macroporous resin for the isolation of tomato saponins. D101 has the highest adsorption efficiency for esculeoside A, with which, $0.25 \mathrm{~g}$ esculeoside A can be obtained from $1 \mathrm{~kg}$ tomato fruit.

\section{Introduction}

Tomato (Lycopersicon esculentum Mill.), a member of the genus Lycopersicon in the family Solanaceae, is native to South America and widely planted in China nowadays. Two structurally complex steroidal saponins were isolated from fresh ripe tomato by Fujiwara from Kumamoto University, Japan in 2004, and named esculeoside $A$ and esculeoside ${ }^{[1]}$.Then, their physicochemical properties, physiological and pharmacological activities were systematically studied, and the results revealed that they were capable of reducing blood lipid, cholesterol, inhibiting cancer cell proliferation, anti-atherosclerosis, anti-oxidation and so on ${ }^{[2]}$. Therefore, esculeoside A and esculeoside B have attracted much attention of experts in cardiovascular diseases all over the world.

Macroporous resin is an organic adsorbent composed of high polymers and was developed in the 1960s. It has an internal three-dimensional stereoscopic pore structure, and good adsorption properties due to the numerous reticular cavities with a large specific surface area ${ }^{[3-4]}$. Macroporous resin can be used for purifying chemical substances from plant materials according to the polarity, relative molecular weight and different solubility of the objects ${ }^{[5]}$. In the present study, the adsorption capacity of 16 different types of macroporous resins to total tomato saponins were measured with spectrophotometry and compared to select the best macroporous resin type, and provide technical support for in-depth study of tomato saponins.

\section{Instruments and Materials}

\section{Materials}

Fresh ripe cherry tomatoes were purchased from the tomato planting base at Chetian Town, Ziyuan County, Guilin. Esculeoside A standard was purchased from Department of Pharmacy, Kumamoto University, Japan, and its mass fraction was 98.9\%, determined by HPLC. Sixteen types of macroporous resins: 1400, AB-8, CA-40, H103, HP-20, HPD100, HPD300, HPD400, HPD600, NKA, NKA2, NKA-9, S-8, Lukang 86021, Lukang DM130 and D101 were purchased from Tianjin 
Zhengtiancheng Resin Factory and Cangzhou Bao'en Adsorbing Material Technology Co., Ltd. Refined pectinase was purchased from Tianjin Lihua Enzyme Technology Co., Ltd. (with batch number: 20090603, food additive).

\section{Instruments and regents}

T-6 spectrophotometer was purchased from Beijing Purkinje General Instrument Co., Ltd.; R-3 rotary evaporator was purchased from BUCHI Labortechnik AG; TL-5.0 desktop centrifuge was purchased from Shanghai Centrifuge Institute Co., Ltd.; TP-2200B electronic balance was purchased from Xiangyi Balance Instrument \& Equipment Co., Ltd.; METTLER AT200 analytical balance was purchased from Shanghai Hanjin Instrument \& Meter Co., Ltd.; vanillin, perchloric acid and glacial acetic acid were all of analytical grade; ethanol and methanol were chemically pure.

\section{Methods and Results}

\section{Pretreatment of macroporous resins}

Columns were filled with each type of macroporous resin $(200 \mathrm{~g})$, which had been soaked with $95 \%$ ethanol for $24 \mathrm{~h}$, eluted with $95 \%$ ethanol at a flow rate of $2 \mathrm{BV} / \mathrm{h}$, till the mixture of the elute and water was not cloudy any more. Then, the columns were washed with deionized water to remove ethanol, and before being washed with $5 \%$ dilute hydrochloric acid at a flow rate of $2 \mathrm{BV} / \mathrm{h}$ and soaked with it for $1 \mathrm{~h}$. Finally, the columns were washed with deionized water till the eluent's $\mathrm{pH}$ value was 7.0.

\section{Pretreatment of samples}

Thirty-four tomato samples were precisely weighed (1 $\mathrm{g}$ each), washed and processed with a juicer. Then, the juice samples were digested with $0.5 \%$ pectinase at $50^{\circ} \mathrm{C}$ for $1.5 \mathrm{~h}$, filtered through gauze before centrifugation. The supernatant was stored in a refrigerator till analys is.

\section{Screening of the optimal macroporous resin}

Three copies of the 16 types of macroporous resin were precisely measured (150ml each copy) and loaded to columns. Then, the pretreated tomato samples were loaded to these columns to measure the adsorption capacity of the resins to total tomato saponins. After that, the columns were washed by 10 volumes of deionized water, and then eluted with $95 \%$ ethanol till the eluent was colorless. Finally, the eluents were concentrated under reduced pressure, vacuum-dried, and the resulting total tomato saponins were accurately weighed.

\section{Measure ment of the content of total saponins in tomato \\ Preparation of standard solution of esculeoside A and crude saponin solution}

5.0mg of esculeoside A standard was precisely weighed (with purity of 98.9\%), diluted with methanol to total volume of $5 \mathrm{ml}$, sonicated to obtain the standard solution of esculeoside A (1.0 $\mathrm{mg} / \mathrm{ml}) .25 .0 \mathrm{mg}$ of crude saponin powder was precisely weighed, diluted with methanol to total volume of $25 \mathrm{ml}$, sonicated, and filtered through microporous membrane to obtain the solution of crude saponins $(1.0 \mathrm{mg} / \mathrm{ml})$.

\section{Selection of measure ment wavelength}

At first, $0.5 \mathrm{ml}$ of standard esculeoside A solution was transferred into a test tube with stopper, incubated in a water bath at $70^{\circ} \mathrm{C}$ till the solvent was evaporated to dryness. Then, $0.2 \mathrm{ml}$ of $5 \%$ vanillin - glacial acetic acid and $0.8 \mathrm{ml}$ of perchloric acid were added to the tube, mixed by shaking, and incubated in a water bath at $60^{\circ} \mathrm{C}$ for $20 \mathrm{~min}$. The tube was taken out of the water bath, allowed to stand at room temperature for $10 \mathrm{~min}$. $10 \mathrm{~min}$ after $5 \mathrm{ml}$ of glacial acetic acid was added, the tube was scanned at $400-700 \mathrm{~nm}$. The maximum absorption was found at 590nm. So, 590nm was considered as the wavelength most suitable for the determination of saponins. 


\section{Establishment of standard curve}

$0.0,0.05,0.10,0.15,0.20,0.25$ and $0.30 \mathrm{ml}$ of standard esculeoside A solutions were precisely measured and transferred to $10 \mathrm{ml}$ tubes with stopper, incubated in a water bath at $70^{\circ} \mathrm{C}$ till the solvent was evaporated. Then, $0.2 \mathrm{ml}$ of newly prepared $5 \%$ vanillin - glacial acetic acid and $0.8 \mathrm{ml}$ of perchloric acid, were added to each tube, stoppered and mixed by shaking. The tubes were incubated in a water bath at $60^{\circ} \mathrm{C}$ for $20 \mathrm{~min}$, allowed to stand at room temperature for $10 \mathrm{~min}$. After $5 \mathrm{ml}$ of glacial acetic acid was added and mixed, the absorbance was read at $590 \mathrm{~nm}$. The stand ard curve was obtained by plotting absorbance on the Y-axis and the weight of esculeoside A on the $\mathrm{X}$-axis. The resulting regression equation was $Y=1.1088 X+0.0492, \mathrm{R}^{2}=0.996(n=6)$, wherein, $X$ is the weight of esculeoside $\mathrm{A}(\mathrm{mg})$ and $Y$ is absorbance. The equation revealed that there was a good linear relationship between absorbance and weight of esculeoside A (in a range of 0.052-0.312 $\mathrm{mg})$.

\section{Verification of experiment stability, accuracy and reproducibility}

$0.2 \mathrm{ml}$ of crude saponins solution prepared above was collected, processed as described in "Establishment of standard curve", and the absorbance was read 1, 2, 3, 4, 5, 6, 7 and 8h later. The RSD was $2.71 \%(n=8)$, indicating that the results were relatively stable within $8 \mathrm{~h}$. Six copies of standard esculeoside A solution $(0.2 \mathrm{ml})$, processed as described in "Establishment of standard curve", and the absorbance was read 1, 2, 3, 4, 5, 6, 7 and $8 \mathrm{~h}$ later. The $R S D$ value was $1.11 \%(n=6)$, suggesting that this method was accurate. Six copies of crude saponin powder (10mg each), diluted with methanol to a total volume of $10 \mathrm{ml} .0 .2 \mathrm{ml}$ of the solution, and its absorbance was measured following the steps described above. The resulting $R S D$ value was $1.14 \%(n=6)$, indicating that the method was repeatable.

\begin{tabular}{clll} 
Table1 The stability, accuracy and reproducibility of the system we developed \\
\hline & Stability & Accuracy & Reproducibility \\
\hline & 0.146 & 0.297 & 0.155 \\
0.148 & 0.290 & 0.156 \\
0.153 & 0.291 & 0.153 \\
Absorbance (A) & 0.155 & 0.299 & 0.157 \\
& 0.16 & 0.293 & 0.156 \\
& 0.149 & 0.292 & 0.152 \\
& 0.152 & & \\
\hline$R S D(\%)$ & 0.151 & & \\
\hline
\end{tabular}

\section{Test on recovery rate of saponins}

Four copies of crude saponins powder samples, in which the esculeoside A content was measured, (about 14\%) were precisely weighed (100 mg each copy). Four copies of esculeoside A standard were also precisely weighed ( $2 \mathrm{mg}$ each) and added to the four copies of crude saponins powder samples. Then, each sample was diluted to $50 \mathrm{ml}$. The peak area was measured to calculate the recovery rate, average recovery rate and $R S D$ value. All the data revealed that the recovery rate and $R S D$ value met the requirements as expected.

Measured weight of esculeoside $\mathrm{A} / \mathrm{mg} \div$ (the weight of esculeoside $\mathrm{A}$ in sample/mg + the weight of esculeoside A added to sample/mg) $\times 100 \%=$ recovery rate 
Table 2 Test on recovery rate

\begin{tabular}{|c|c|c|c|c|c|c|}
\hline $\begin{array}{l}\text { Crude saponins } \\
\text { (mg) }\end{array}$ & $\begin{array}{l}\text { Esculeoside A in } \\
\text { crude sample (mg) }\end{array}$ & $\begin{array}{l}\text { Esculeoside } \\
\text { added to sample } \\
(\mathrm{mg})\end{array}$ & $\begin{array}{l}\text { Measured weight of } \\
\text { esculeoside A (mg) }\end{array}$ & $\begin{array}{l}\text { Recovery } \\
\text { rate }(\%)\end{array}$ & $\begin{array}{l}\text { Mean of } \\
\text { recovery } \\
\text { rate }(\%)\end{array}$ & $\begin{array}{l}\mathrm{RSD} \\
(\%)\end{array}$ \\
\hline 101.92 & 11.19 & 2.10 & 12.74 & 95.86 & \multirow{4}{*}{96.03} & \multirow{4}{*}{0.41} \\
\hline 101.30 & 11.12 & 1.98 & 12.66 & 96.65 & & \\
\hline 101.20 & 11.11 & 1.95 & 12.48 & 95.58 & & \\
\hline 100.70 & 11.05 & 2.28 & 12.80 & 96.02 & & \\
\hline
\end{tabular}

Table 3 Adsorption capacity of different types of macroporous resins to esculeoside A

\begin{tabular}{|c|c|c|c|c|}
\hline No. & Macroporous resin & Weight of crude saponins (g) & Weight of esculeoside A (g) & $\begin{array}{l}\text { Percentage of esculeoside } \\
\text { A }(\%)\end{array}$ \\
\hline 1 & D-101 & 1.90 & 0.2381 & 12.53 \\
\hline 2 & AB-8 & 2.02 & 0.2117 & 10.48 \\
\hline 3 & HP-20 & 2.17 & 0.2114 & 9.74 \\
\hline 4 & HPD-600 & 1.84 & 0.2077 & 11.29 \\
\hline 5 & HPD-400 & 1.79 & 0.2042 & 11.41 \\
\hline 6 & Lukang-86021 & 1.66 & 0.2037 & 12.27 \\
\hline 7 & HPD-300 & 2.04 & 0.2034 & 9.97 \\
\hline 8 & HPD-100 & 1.97 & 0.2013 & 10.22 \\
\hline 9 & CA-40 & 1.73 & 0.1953 & 11.29 \\
\hline 10 & $\mathrm{H}-103$ & 2.52 & 0.1887 & 7.49 \\
\hline 11 & NKA & 1.94 & 0.1862 & 9.60 \\
\hline 12 & S-8 & 1.12 & 0.1858 & 16.59 \\
\hline 13 & Lukang-DM 130 & 1.81 & 0.1855 & 10.25 \\
\hline 14 & NKA-9 & 0.99 & 0.1817 & 18.35 \\
\hline 15 & 1400 & 1.89 & 0.1724 & 9.12 \\
\hline 16 & NKA-2 & 0.90 & 0.0140 & 1.56 \\
\hline
\end{tabular}

\section{Determination of the content of total saponins in samples}

The standard esculeoside A solution and tomato sample solution prepared above were pipetted $\left(0.5 \mathrm{ml}\right.$ each) and transferred to test tubes with stopper, incubated in a water bath at $70^{\circ} \mathrm{C}$ till the solvent was evaporated to dryness. Then, $0.2 \mathrm{ml}$ of $5 \%$ vanillin - glacial acetic acid and $0.8 \mathrm{ml}$ of perchloric acid were added to each tube, mixed by shaking, incubated in a water bath at $60^{\circ} \mathrm{C}$ for $20 \mathrm{~min}$. The reaction was stopped by incubating in ice for $10 \mathrm{~min}$. Ten minutes after $5 \mathrm{ml}$ of glacial acetic acid was added to each tube, the absorbance was read at 590nm. Finally, the content of total tomato saponins in samples was calculated from absorbance according to the standard curve obtained above. 


\section{Results and Analysis}

As shown in Table 3, among all types of macroporous resin, D-101 had the best absorption capacity to esculeoside A, followed by AB-8. NKA-9 was most specific to esculeoside A and thus the resulting percentage of esculeoside A was the largest, although it had a lower absorption capacity. In addition, D-101 and AB- 8 were two types of macroporous resin most commonly used in previous studies for the extraction of saponins, so there is a need to determine the optimal parameters for saponin extraction.

\section{Discussion}

Currently, saponins in ginseng, bitter gourd, yam and other plants have been studied a lot, but saponins in tomato has been rarely reported. It has been proven that saponins have a variety of physiological and pharmacological activity ${ }^{[6-7]}$. We have found that tomato saponins has a unique role in lipid-lowering and anti-atherosclerosis. It can regulate lipid metabolism, lower blood viscosity, increase the capacity of oxidative free radical scavenging, reduce lipid peroxide, which prevents and suppresses the formation of atherosclerosis ${ }^{[8]}$. Moreover, the water extract of tomato saponins can effectively regulate lipid metabolism in human bodies, and thus can be used for the treatment of hyperlipidemia ${ }^{[9]}$. Macroporous resins have been widely used for the isolation and purification of saponins, alkaloids, flavonoids, peptides, carbohydrates and other chemicals ${ }^{[10]}$. In this study, the macroporous resin type most suitable for the isolation of tomato saponins was determined, which will provide a technical support for in-depth study of saponins.

Tomato saponins have no absorption in UV region. It will need a capital investment in instruments using evaporative light scattering derector to detect them. Vanillin colorimetry is simple, low-cost, and reliable, but the results are greatly affected by temperature and reaction time. So the reaction conditions should be strictly controlled and three or more repetitions should be prepared when vanillin colorimetry is adopted for the measurement of tomato saponins.

\section{Acknowledgements}

This work was supported by Agricultural science and Technology Achievements Transformation Fund Project(2014GB2E100274); Fund of Guangxi Key Laboratory of Functional Phytochemicals Research and Utilization (ZRJJ2012-6); S upported by Bagui Scholar Program of Guangxi (Guangxi Institute of Botany).

\section{References}

[1] Yukio Fujiwara, Naoko Kiyota, Masaharu Hori, Sayaka Matsushita, Yoko Iijima, Koh Aoki, Daisuke Shibata, Motohiro Takeya, Tsuyoshi Ikeda, Toshihiro Nohara, Ryoji Nagai. American Heart Association, 2007, (27): 2400-2406.

[2] Friedman M , Levin CE, Lee SU , Kim HJ , Lee IS , Byun JO , Kozukue N. Agricultural and Food Chemistry, 2009, (57): 5727-5733.

[3] Maotian Wang, Shan Pei. Chemical Industry Press , 2004: 100-105. (In Chinese)

[4]Chenghe Sun, Yingping Wang, Shiying Zhao Special Wild Economic Animal and Plant Research, 2008, (2): 55-57. (In Chinese)

[5] Xiaomin Li , Xuan Wu, Zhongliang Xiong . Journal of Chinese Medicinal Materials, 2009, 10(32): 1613 -1615.(In Chinese)

[6] Ting Jin . Food Engineering, 2008, (1): 19-21.(In Chinese) 
[7] Yongxia Tao , Jihua Duan . Academic Periodical of Farm Products Processing, 2007, (6): 84-85. (In Chinese)

[8] Jianzhang Wu, CiyuLi, Jinlei Liu . Food Research and Development, 2011, (11): 131-134. (In Chinese)

[9] Jianzhang Wu, CiyuLi, Jinlei Liu . Food Research and Development, 2011, (3): 167-170.(In Chinese)

[10] Haixia Zhao, Jian Tong, China Food Additives, 2009, (7): 131-134. (In Chinese) 